\title{
Minireview
}

\section{The transcriptome of human monocyte subsets begins to emerge}

\author{
Fernando O Martinez
}

Address: Sir William Dunn School of Pathology, University of Oxford, Oxford, OX1 3RE, UK. Email: fernando.martinezestrada@path.ox.ac.uk

\begin{abstract}
Human monocytes can be divided into subsets according to their expression or lack of the cell-surface antigen CD16. In papers published recently in the Journal of Proteome Research and in BMC Genomics, two groups publish independent transcriptome analyses of $\mathrm{CD}^{-} 6^{+}$and $\mathrm{CD}^{-} 6^{-}$monocytes, with revealing results.
\end{abstract}

See research article http://www.biomedcentral.com/1471-2164/10/403

Monocytes are a heterogeneous group of cells constituting $5-10 \%$ of the total white blood cells in humans. They originate in the bone marrow, circulate in the bloodstream and enter tissues, where they differentiate into macrophages, either to replenish the stock of tissue macrophages or to contribute to an inflammatory response to infection. Monocytes can remain in the circulation for up to 72 hours, after which, if they have not been activated, they die and are removed.

The heterogeneity of monocytes was noticed soon after their definition, and it includes differences in density, production of reactive oxygen species, antigen-presenting capacity, maturation status, and phagocytic and adhesive properties. In 1989, Ziegler-Heitbrock and colleagues [1] noticed that human monocytes can be divided into three main populations according to their expression of the cellsurface antigens CD16 (Fcy receptor III) and CD14 (a receptor for bacterial lipopolysaccharide (LPS)). The CD16 Fcy receptor is a relatively low-affinity receptor for the Fc portion of IgG antibodies in complex with their antigens, and stimulates the monocyte to take up antibody-antigen complexes by phagocytosis and thus remove them from the circulation. CD14 is essential for the recognition of bacterial LPS present in Gram-negative bacteria, which include many common pathogens. CD14 acts as pattern recognition protein which accepts LPS from LPS-Binding protein. To elicit the endotoxin cellular response the CD14LPS complex interacts with various Toll like receptors (TLR) including TLR4-MD2 (myeloid differentiation factor-2), TLR2/TLR6 and TLR2/TLR1.

The preponderant phenotypes are monocytes expressing CD14 but not CD16 (CD14 $\left.{ }^{++} / \mathrm{CD}_{16}\right)$ ) and those expressing CD16 and low CD14 $\left(\mathrm{CD}_{14}+\mathrm{CD}^{+} 6^{+}\right)$, and there is also a smaller subpopulation of monocytes expressing CD14 and
CD16 $\left(\mathrm{CD}_{14}{ }^{++} / \mathrm{CD} 16^{+}\right)$. All monocytes expressing CD16 (hereafter referred to as $\mathrm{CD}_{16} 6^{+}$) are considered to be proinflammatory, as they are better than $\mathrm{CD}^{-} 6^{-}$monocytes (the $\mathrm{CD}_{14}{ }^{++} / \mathrm{CD}_{16} 6^{-}$subset) at producing the cytokines tumor necrosis factor (TNFa), interleukin (IL)-6 and IL-10 in response to microbial-associated molecular patterns $[2,3]$. In addition, $\mathrm{CD}_{16}{ }^{+}$cells are better at phagocytosis and at producing microbicidal reactive nitrogen intermediates than are CD16- cells. The CD16- subset, which is the predominant monocyte population in the circulation in a healthy person in the absence of infection, has more effective antimicrobial capacity and is more efficient at producing microbicidal reactive oxygen species. Additional functional differences are summarized in Figure 1.

The ratio of $\mathrm{CD} 16^{+}$to $\mathrm{CD} 16^{-}$monocytes changes greatly in disease. Results accumulated over the past decade suggest that the $\mathrm{CD}_{16} 6^{+}$subset is expanded in a vast number of inflammatory diseases, irrespective of their etiology. A higher $\mathrm{CD}_{16} 6^{+}$to $\mathrm{CD} 16^{-}$ratio (as compared with that typical of a healthy person) has been found in Crohn's disease [4], rheumatoid arthritis, asthma and sepsis, among other diseases [2]. In hypoxic episodes, such as those caused by myocardial infarction and stroke, higher levels of CD16- monocytes (but not $\mathrm{CD} 16^{+}$) correlate with disease severity and poor outcome. The increase in numbers of $\mathrm{CD}_{16} 6^{+}$monocytes are such a general feature of disease that, although it can indicate severity and outcome, it cannot suggest a specific diagnosis. Various explanations have been proposed for the increase in circulating $\mathrm{CD}_{16}{ }^{+}$cells in inflammatory disease: the maturation of $\mathrm{CD}^{-} 6^{-}$cells into $\mathrm{CD} 16^{+}$cells; the increased movement of CD16- monocytes out of the blood vessels into tissues; and even the stimulation of a putative $\mathrm{CD}_{16} 6^{+}$ monocyte developmental pathway.

Investigating monocyte subset transcriptomes In the past decade, we have learned a great deal about the functional and phenotypic differences between the monocyte subsets, with most investigations looking at wellestablished molecules or defined monocytic properties. In the late 1990 s and early 2000s, with the sequencing of the human and other genomes, microarray technology for studying the transcriptomes of human cells became available, and in the past few years this methodology has been used to investigate the detailed differences between 


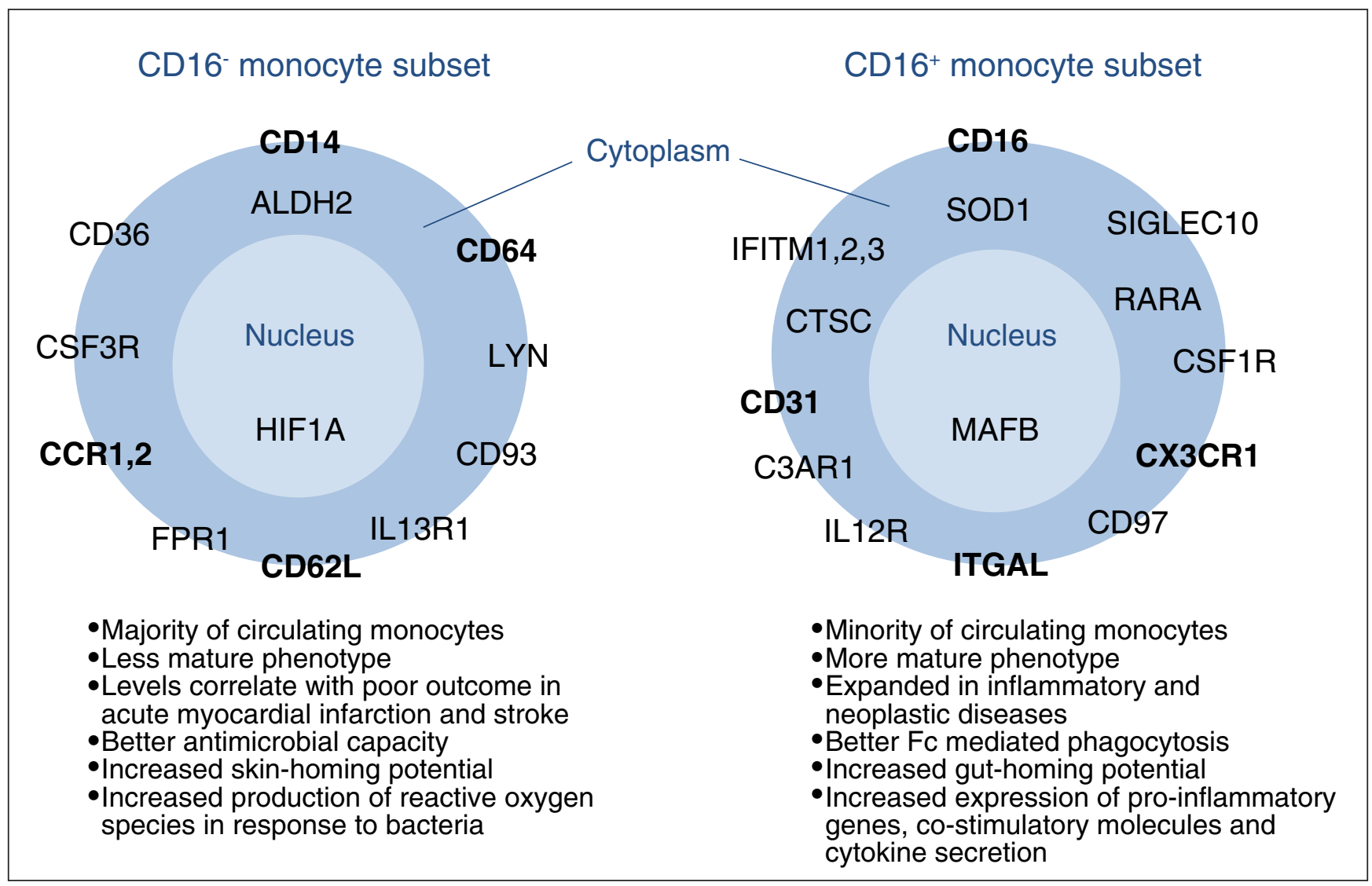

Figure 1

Summary of selected established and proposed functional differences between human CD16- and CD16+ monocyte subsets. Genes expressed at a high level in one subset but not in the other are indicated. Gene labels are positioned according to the location of the protein in the cell - in the plasma membrane, the cytoplasm or the nucleus. Several studies have previously confirmed high levels of expression in human $\mathrm{CD} 16^{+}$monocytes of the genes for the chemokine receptor CX3CR1, the integrin alpha chain ITGAL and the adhesion molecule CD31 and strong expression in human CD16- monocytes of genes for the adhesion molecule CD62L, the high-affinity Fc receptor for IgG (CD64), and the chemokine receptors CCR1 and CCR2. The new studies add many candidates to the monocyte subsets markers list, a selection of which is represented in the figure. Previously confirmed markers are in bold. A list of differentially expressed genes found by both $[6,7]$ is given in Additional data file 1. Other abbreviations of markers: ALDH2, aldehyde dehydrogenase 2 family; C3AR1, complement component 3a receptor 1; CD26, dipeptidyl-peptidase 4; CD93, CD93 molecule; CD99, CD99 molecule; CSF1R, colony stimulating factor 1 receptor; CSF3R, colony stimulating factor 3 receptor; CTSC, cathepsin C; CD97, CD97 molecule; FPR1, formyl peptide receptor 1; HIF1A, hypoxia inducible factor 1, alpha subunit (basic helix-loop-helix transcription factor); IFITM1-3, Interferon-induced transmembrane protein 1, 2 and 3; IL12R, Interleukin 12 receptor; IL13R1, Interleukin 13 receptor 1, LYN, v-yes-1 Yamaguchi sarcoma viral related oncogene homolog; MAFB, v-maf musculoaponeurotic fibrosarcoma oncogene homolog B (avian); RARA, retinoic acid receptor alpha subunit; SIGLEC10, sialic acid binding Ig-like lectin 10; SOD1, soluble superoxide dismutase 1.

human monocyte subsets. Microarrays are now commercially available that allow investigation of the expression of all known human genes and as-yet-unidentified transcribed sequences.

At least three microarray studies focused on monocyte subsets have been published. In 2007, Mobley et al. [5] studied the differences in gene expression between monocyte subsets using the Affymetrix Human 133A 2.0 array, which analyzes the expression level of 18,400 transcripts and variants, including 14,500 well characterized human genes. In this study [5], human peripheral mononuclear cells
(PBMCs) were isolated from total blood using a Ficoll gradient and were subsequently incubated with paramagnetic beads conjugated with specific antibodies to deplete natural killer (NK) cells, T lymphocytes and B lymphocytes. After separation the resultant fraction, highly enriched in monocytes, was then split to isolate $\mathrm{CD}_{16} 6^{+}$and $\mathrm{CD} 16^{-}$monocytes. $\mathrm{CD} 16^{+}$monocytes were positively selected using an anti-CD16 antibody. To isolate CD16- monocytes the authors [5] used magnetic beads conjugated with antibodies against the cell adhesion molecule CD62L, also known as L-selectin. This molecule is found on most peripheral leukocytes and divides human 
monocytes into two fractions. The $\mathrm{CD} 62 \mathrm{~L}^{+}$monocytes are mostly $\mathrm{CD} 14^{++} \mathrm{CD}^{-} 6^{-}$, whereas the $\mathrm{CD} 62 \mathrm{~L}^{-}$monocytes are $\mathrm{CD} 14^{+} \mathrm{CD} 16^{+}$. This procedure of isolation differs from that used in other studies $[6,7]$.

Mobley et al. [5] provided a selective list of genes differentially expressed in $\mathrm{CD}_{16} 6^{+}$and $\mathrm{CD} 16^{-}$monocytes. The list includes 15 genes highly expressed in CD14 ${ }^{++} \mathrm{CD} 16^{-}$monocytes and 19 genes highly expressed in $\mathrm{CD} 14^{+} \mathrm{CD} 16^{+}$monocytes. They found that $\mathrm{CD} 16^{+}$ monocytes have higher mRNA levels of known subset biomarkers such as CD16 and the chemokine receptor $\mathrm{CX}_{3} \mathrm{CR} 1$, but also new markers such as the colonystimulating factor 1 receptor (CSF1R), the receptor for macrophage colony-stimulating factor (MCSF) and the complement component factors $\mathrm{C}_{1} \mathrm{QA}, \mathrm{C} 1 \mathrm{QB}$ and $\mathrm{C}_{3}$. MCSF is a potent maturation signal for monocytes and a survival and proliferative factor for macrophages and their precursors, and it is required for the development of many types of tissue macrophage.

In CD16- monocytes Mobley et al. [5] found higher expression of CD14 and the chemokine receptor CCR2. Their data show for the first time higher expression in CD16- monocytes of the colony-stimulating factor 3 receptor $\left(\mathrm{CSF}_{3} \mathrm{R}\right)$. Colony-stimulating factor 3 ( $\mathrm{CSF}_{3}$; also called GMCSF) is another important maturation factor for monocytes and is also a maturation factor for granulocytes. In this study [5], no details about the total number of differentially expressed genes or ways of accessing the dataset are given.

Recently, two other investigations have been published, by Zhao et al. [6] in the Journal of Proteome Research and Ancuta et al. [7] in BMC Genomics. Both of these comply with MIAME (Minimal Information about Microarray Experiments) requirements and provide their data. In a well executed study, Zhao et al. [6] used magnetic beads carrying anti-CD16 antibodies to isolate $\mathrm{CD} 16^{+}$monocytes from PBMCs that had been depleted of NK cells and neutrophils. CD16- monocytes were isolated from the CD16-negative fraction using antiCD14 beads. $\mathrm{CD}_{16}{ }^{+}$and $\mathrm{CD} 16^{-}$monocytes were also isolated by fluorescence-activated cell sorting (FACS) from total monocytes purified by CD14-positive selection. The transcriptomes of the two subsets were then defined using the Illumina BeadArray HG-6v2. In this study [6], 521 genes were scored as differentially expressed between the subsets: 305 characterized the CD16- subset and 216 the CD16 ${ }^{+}$subset. The authors also investigated differences between subsets at the protein level. The proteomic approach showed that out of 1,006 proteins robustly expressed, 235 were differentially expressed between the subsets: of these, 123 proteins characterized the $\mathrm{CD}_{16} 6^{+}$monocytes and 112 the CD16monocytes.
The three most represented Gene Ontology (GO) categories for differentially expressed mRNAs were cellular growth and proliferation, cell death, and metabolism; for differentially expressed proteins they were cell death, metabolism, and cellular assembly [6]. Known subset biomarkers such as $\mathrm{CD} 16$ and $\mathrm{CD} 14$, the chemokine receptors $\mathrm{CX}_{3} \mathrm{CR} 1$ and CCR2, the integrin alpha $_{\mathrm{L}}$ (ITGAL) and alpha (ITGAM) chains, and the leukocyte adhesion molecule CD62L were among the genes modulated at the mRNA level (Figure 1), of which CD16, ITGAL and ITGAM were also identified at the protein level.

In addition to $\mathrm{CD} 16$ itself, in the $\mathrm{CD}_{1} 6^{+}$subset the authors [6] found overexpression of genes that participate in Fc $\gamma$ R-mediated phagocytosis. They confirmed higher mRNA levels for heme oxygenase 1 (HMOX1), villin 2 (VIL2), hematopoietic cell kinase (HCK) and the tyrosine protein kinase Lyn (LYN). At the protein level they confirmed higher expression of actinrelated protein 2/3 complex (ARP2 and ARP3), $\mathrm{HCK}$ and LYN. In the CD16- subset they found instead overexpression of genes involved in antimicrobial functions. In this subset were confirmed higher mRNA levels of myeloperoxidase (MPO), lysozyme C (LYZ), Protein S100-A9 (S100A9), eosinophil cationic protein (RNASE3) and phospholipase B domain containing 1 (PLBD1, also FLJ22662). Higher protein levels were confirmed for cathepsin G (CTSG), MPO, LYZ and S10oA9. Among other interesting conclusions, this study [6] clearly showed that mRNA and protein levels do not always correlate in the subsets. Thus, mRNA levels seem to represent the potential, more than the actual, functional capacity of the monocytes.

Ancuta et al. [7] isolated total monocytes from PBMCs by negative selection using magnetic beads to remove the other cell types; the $\mathrm{CD} 6^{+}$monocytes were subsequently isolated by positive selection using anti-CD16 magnetic beads. The transcriptomes were defined using the Affymetrix HGU133 microarray. Applying rigorous and exemplary statistics, the authors defined a set of 361 genes that distinguish $\mathrm{CD} 16^{+}$ from $\mathrm{CD}_{16}{ }^{-}$monocytes: these comprise 172 genes and unknown transcribed sequences that are highly expressed in $\mathrm{CD} 6^{+}$monocytes, and 189 genes and unknown transcribed sequences highly expressed by CD16- monocytes. Applying more stringent statistics they provide a shortlist of 61 genes, of which 30 transcripts are upregulated in $\mathrm{CD}_{16} 6^{+}$and 31 in CD16- monocytes.

Classifying their larger dataset of differentially expressed genes, Ancuta et al. [7] found over-representation in key GO categories, including immune response, inflammation, metabolism and stress response, cell cycle, proliferation and differentiation. They also found over-representation of more informative functional subcategories: for example, cytokines, chemokines and complement (both ligands and 
receptors); signaling and signal transduction; cytoskeleton; and transcription factors.

Ancuta et al. [7], like the other authors [5,6], confirmed expected subset biomarkers. All three authors found at the mRNA level, and Ancuta et al. [7] at the protein level, that $\mathrm{CD}^{+} 6^{+}$monocytes express higher levels of CSFR1. In agreement with Zhao et al. [6], Ancuta et al. [7] found that CD16 ${ }^{+}$monocytes have higher mRNA levels of the IL-12 receptor 1 (IL12RB1). IL-12 is a cytokine produced by activated monocytes, macrophages and dendritic cells and is essential for resistance to bacterial and intracellular parasite infection. In addition they [7] found differential expression of the complement component $\mathrm{C}_{3}$ receptor 1 (C3AR1) on $\mathrm{CD}_{16}{ }^{+}$monocytes. This receptor recognizes the chemotactic and inflammatory peptide anaphylatoxin $\mathrm{C}_{3}$. $\mathrm{C}_{3} \mathrm{a}$ is one of the products of the proteolytic cleavage of complement component $\mathrm{C}_{3}$, which was found to be highly expressed by $\mathrm{CD} 16^{+}$monocytes by Mobley et al. [5].

This gene-expression pattern functionally contrasts with the higher mRNA levels in CD16- monocytes of the IL-13 receptor 1 (IL13RA1), found by Zhao et al. [6] and Ancuta et al. [7]. IL13RA1 is a subunit of one of the receptors for IL-4 and IL-13. These cytokines induce the 'alternative' activation of monocytes and macrophages, enhancing macrophage capacity for fluid-phase pinocytosis and endocytosis, and inducing giant cell formation and specific gene signatures. Zhao et al. [6] and Ancuta et al. [7] found at the mRNA level, and Ancuta et al. [7] confirmed at the protein level, that CD16- monocytes express higher levels of $C D 93$, the receptor for complement component C1q1 (also called $\mathrm{C} 1 \mathrm{QR} 1$ ). This receptor is part of a larger receptor complex for C1q complement factor, mannosebinding lectin (MBL2) and pulmonary surfactant protein A (SPA), all proteins that enhance phagocytosis in monocytes.

All three groups [5-7] found that colony-stimulating factor 3 receptor (CSF3R) is highly expressed at the mRNA level in CD16- monocytes and Ancuta et al. [7] demonstrated it at the protein level. The contrasting expression of the colony-stimulating factor receptors $\mathrm{CSF} 1 \mathrm{R}$ and $\mathrm{CFS}_{3} \mathrm{R}$ increases the repertoire of confirmed membrane markers that characterize human monocyte subsets. These findings reveal an unnoticed compensatory loop in the activation balance and perhaps even origin of the subset phenotypes. $\mathrm{CD} 6^{+}$pro-inflammatory monocytes express higher levels of MCSF receptor (CFS1R); MCSF induces macrophages with less pro-inflammatory capacity than GMCSF; and the GMCSF receptor ( $\left.\mathrm{CFS}_{3} \mathrm{R}\right)$ is in turn highly expressed in CD16- classical monocytes [8].

The most recent studies [6,7], using model isolation methods and excellent statistics, provide a set of transcripts that characterize each subset. These gene lists are extensive enough to allow a robust comparison between them. I have determined the overlap in gene symbols, although there are more accurate ways of doing this type of comparison, for instance taking into account the probe sequences used in the arrays, as exemplified by Barnes et al. [9]. For the comparison, I eliminated all genes without gene symbols or duplicated from the lists of all genes up- and downregulated in monocyte subsets provided in Ancuta et al. [7] and in Zhao et al. (Table $\mathrm{S}_{3}$ of [6]). This procedure yielded 318 unique gene symbols from Ancuta et al. [7] and 434 unique gene symbols from Zhao et al. [7]. Merging these two lists showed that 145 genes were identified by both studies, representing $24 \%$ of a total of the 752 different genes associated with monocyte subset differences (Figure 2a). A full list of these genes is given in Additional data file 1. The rest of the differentially expressed genes identified by the two studies were not shared.

The discrepancy between the studies may be due to differences in cell isolation methodology and the purity of the cell populations isolated, the use of negative versus positive selection, and the microarray methodology, among other factors. In fact, although Barnes et al. [9] and others have demonstrated that Affymetrix and Illumina platforms yield highly comparable data, especially for genes predicted to be differentially expressed, the platforms use different amounts of total RNA for the hybridization and different probes to identify the genes and even distinct solid supports for the probes [9]. Further transcriptomic and proteomic studies will clarify the discrepancies found so far and will shed light on this topic.

\section{Comparison with macrophage maturation transcriptomes}

It has been hypothesized that the difference between monocyte subsets is due to a difference in stage of maturation [2]. This could be directly influenced (at least in part) by levels of MCSF in the environment and by the differential expression in the subsets of the receptor for MCSF. It is therefore of interest to compare the genes that distinguish the monocyte subsets $[6,7]$ with those involved in the maturation of total human monocytes induced by MCSF in vitro and in macrophage activation (approximately 3,530 genes in total) [8]. Representatives of the latter category of cells are macrophages stimulated with a combination of interferon gamma (INF- $\gamma$ ) and LPS, which induces a classical pro-inflammatory and antimicrobial phenotype in macrophages, and IL-4, which as previously mentioned induces an alternative type of activation. This comparison shows that out of the 434 genes extracted as differentially expressed from [6], 190 are also contained in the maturation/activation gene set, and out of the 318 genes identified from [7], 180 overlap with the maturation/activation data. 
(a)

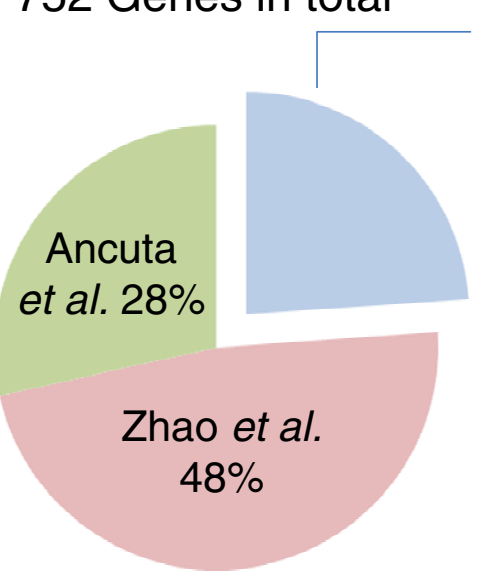

(b)

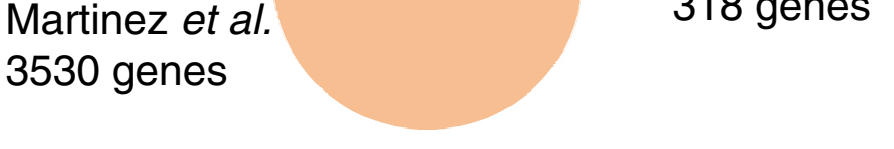

\section{Intersection} $24 \%$

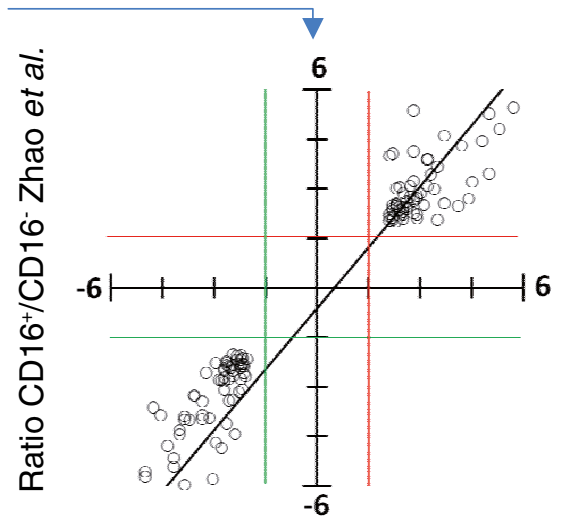

Ratio CD16 ${ }^{+}$CD16- Ancuta et al.

\section{Intersection 86 genes}

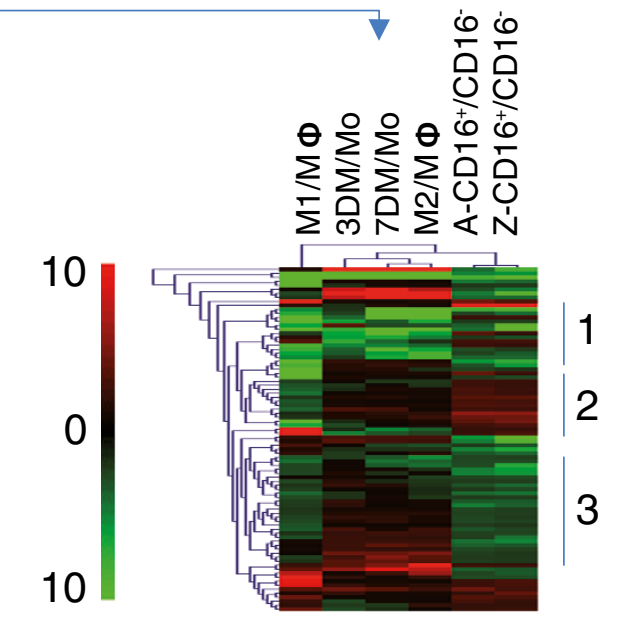

Figure 2

Direct comparison between high-throughput genomic studies hints at a complex interplay between genes as the basis for the differences between monocyte subsets. (a) The overlap between differentially expressed genes identified by Zhao et al. [6] and Ancuta et al. [7] was determined after eliminating all genes without gene symbols or duplicated. Merging the two studies we find that the number of genes differentially expressed between monocyte subsets amounts to 752 (100\%). Of these $24 \%$ (145 genes) are genes detected in both studies; $48 \%$ of the remaining genes are detected as differentially expressed by [6] and $28 \%$ by [7]. A scatter-plot of the fold expression difference in CD16 ${ }^{+}$compared with CD16- monocytes shows correlation of the values in the two studies. Red and green cutoff lines extend along the values 1.5 and -1.5 . The upper right quadrant and the lower left quadrant show genes with similar fold differences between the studies. (b) Overlap between the monocyte subset gene lists and a dataset of genes involved in human monocyte maturation induced by MCSF and macrophage activation induced by a combination of LPS and IFN-y (M1) or IL-4 (M2) [8]. This comparison shows that 190 out of the 434 genes selected from [6], and 180 out of the 318 genes selected from [7], are also contained in the maturation/activation gene set. The overlap of the three lists amounts to 86 genes. A hierarchical clustering of these 86 genes shows that a proportion of them are regulated by MCSF and contrastingly regulated by the combination of IFN-y and LPS used in [8] to drive macrophages towards classical activation. The differences in expression between subsets only partially correlate with the MCSF gene expression pattern. The tree can be divided in three main clusters (right). Cluster 1 shows genes downregulated by MCSF stimulation in total monocytes and whose levels are lower in CD16 monocytes. Cluster 2 shows genes induced by MCSF stimulation that are highly expressed by CD16 ${ }^{+}$monocytes. These two clusters support the hypothesis that part of the differences between subsets correlates with an MCSF responsive phenotype for CD $16^{+}$monocytes. However, the behaviors of the genes in cluster 3 do not correlate with those of MCSF stimulation, and instead correlates with the inflammatory profile induced by the combination of IFN-y and LPS used in [8] to drive macrophages towards classical activation. A list of the 86 overlapping genes is given in Additional data file 2. Other abbreviations used: M $\Phi$, macrophage; Mo, monocytes; 3DM, Monocytes stimulated for three days with MCSF equivalent to $3^{\text {rd }}$ day macrophages; 7DM, Monocytes stimulated for seven days with MCSF equivalent to $7^{\text {th }}$ day macrophages. In the figure the ratio of RNA expression between given categories is indicated by the forward slash or stroke symbol "/". A-CD16+/CD16-, Ratio of RNA expression levels of CD16+ vs CD16- monocytes provided by Ancuta et al.[7]; Z-CD16"/CD16-, Ratio of $\mathrm{CD}^{+} 6^{+}$vs CD16- monocytes provided by Zhao et al.[6]. 
Of the 145 differentially expressed genes found by both studies [6,7], a total of 86 (59\% of 145) were shared with the maturation/activation gene set (Figure 2b; Additional data file 2). Hierarchical clustering of the expression ratio of these 86 genes shows, however, that only a proportion of them are regulated by MCSF. Of the set of genes regulated by MCSF (data from [6-8]), clusters 1 and 2 contain genes whose levels correlate with the ratio in $\mathrm{CD}_{16} 6^{+}$versus $\mathrm{CD} 16^{-}$ monocytes (Figure 2b). These two clusters support the view that $\mathrm{CD}_{16}{ }^{+}$monocytes are more responsive to MCSF. However, cluster 3 shows that not all genes induced by MCSF are higher in $\mathrm{CD}_{16}{ }^{+}$monocytes than in $\mathrm{CD}_{16}^{-}$ monocytes. In addition, these genes seem to correlate with the pattern induced by interferon gamma (IFN- $\gamma$ ) and bacterial LPS when these cytokines are used to drive macrophages toward classical activation. The correlation between $\mathrm{CD}_{16}{ }^{+} / \mathrm{CD} 16^{-}$profiles and those of inflammatory stimuli reinforces the suggestion that not only MCSF but also other factors may contribute to differences between monocyte subsets $[2,6,7]$.

Many issues about monocyte subsets await clarification. The origin of the subsets and the basis and meaning of the fluctuations in their numbers in health and disease remain unexplained. The therapeutic potential of depleting specific subsets has been assessed in several studies, with the aim of reducing inflammation. Several therapies for autoinflammatory diseases decrease the numbers of $\mathrm{CD} 16^{+}$ monocytes in the blood; for example, glucocorticoids reduce the number of $\mathrm{CD} 16^{+}$monocytes by $95 \%$ after 5 days. However their systemic effects are many, including potent immunosuppression, osteoporosis and hypertension [10]. Monocyte depletion by apheresis using an adsorptive Adacolumn seems more selective, and the results from early trials of this device in Crohn's disease are promising [4]. Understanding the role and full potential of the monocyte subsets in the inflammatory response will be essential for creating novel and directed therapeutic approaches.

\section{Additional data files}

Additional data are provided with this article online. Additional data file 1 lists the genes differentially expressed by $\mathrm{CD}_{16} 6^{+}$and $\mathrm{CD}_{16} 6^{-}$monocytes that were detected in common by Zhao et al. [6] and Ancuta et al. [7]. Additional data file 2 lists genes differentially expressed by $\mathrm{CD}_{16}{ }^{+}$and
CD16- monocytes (from [6,7]) in common with a dataset of genes expressed during monocyte maturation (3 days and 7 days after stimulation with MCSF) and activation by the classical (M1) and alternative (M2) pathway [8].

\section{Acknowledgements}

I thank Wong Siew Cheng and Petronela Ancuta for reviewing the manuscript and providing helpful suggestions, and Megan Kerr and Janet Digby for proofreading.

\section{References}

1. Passlick B, Flieger D, Ziegler-Heitbrock HW: Identification and characterization of a novel monocyte subpopulation in human peripheral blood. Blood 1989, 74:2527-2534.

2. Ziegler-Heitbrock L: The $\mathrm{CD}^{+}{ }^{+} \mathrm{CD}^{+} 6^{+}$blood monocytes: their role in infection and inflammation. J Leukoc Biol 2007, 81:584-592.

3. Tacke F, Randolph GJ: Migratory fate and differentiation of blood monocyte subsets. Immunobiology 2006, 211:609618.

4. Hanai H, lida T, Takeuchi K, Watanabe F, Yamada M, Kikuyama M, Maruyama Y, Iwaoka Y, Hirayama K, Nagata S, Takai K: Adsorptive depletion of elevated proinflammatory CD14+CD16+DR ${ }^{++}$monocytes in patients with inflammatory bowel disease. Am J Gastroenterol 2008, 103:1210-1216.

5. Mobley JL, Leininger M, Madore S, Baginski TJ, Renkiewicz R: Genetic evidence of a functional monocyte dichotomy. Inflammation 2007, 30:189-197.

6. Zhao C, Zhang H, Wong WC, Sem X, Han H, Ong SM, Tan YC, Yeap WH, Gan CS, Ng KQ, Koh MB, Kourilsky P, Sze SK, Wong SC: Identification of novel functional differences in monocyte subsets using proteomic and transcriptomic methods. J Proteome Res 2009, 8:4028-4038.

7. Ancuta P, Liu KY, Misra V, Wacleche VS, Gosselin A, Zhou X, Gabuzda D: Transcriptional profiling reveals developmental relationship and distinct biological functions of CD16 ${ }^{+}$ and CD16- monocyte subsets. BMC Genomics 2009, 10:403.

8. Martinez FO, Gordon S, Locati M, Mantovani A: Transcriptional profiling of the human monocyte-to-macrophage differentiation and polarization: new molecules and patterns of gene expression. J Immunol 2006, 177:73037311.

9. Barnes M, Freudenberg J, Thompson S, Aronow B, Pavlidis P: Experimental comparison and cross-validation of the Affymetrix and Illumina gene expression analysis platforms. Nucleic Acids Res 2005, 33:5914-5923.

10. Dayyani F, Belge KU, Frankenberger M, Mack M, Berki T, Ziegler-Heitbrock L: Mechanism of glucocorticoid-induced depletion of human CD14+CD16 ${ }^{+}$monocytes. J Leukoc Biol 2003, 74:33-39.

Published: 23 December 2009

doi:10.1186/jbiol206

(C) 2009 BioMed Central Ltd 